\title{
A Convex Optimization Approach for the Design of Supergain Electrically Small Antenna and Rectenna Arrays Comprising Parasitic Reactively Loaded Elements
}

\author{
Apostolos Georgiadis, Senior Member, IEEE and Nuno Borges Carvalho, Fellow, IEEE
}

\begin{abstract}
A convex optimization formulation is provided for antenna arrays comprising reactively loaded parasitic elements. The objective function consists of maximizing the array gain, while constraints on the admittance are provided in order to properly account for reactive loads. Topologies with two and three electrically small dipole arrays comprising one fed element and one or two parasitic elements respectively are considered and the conditions for obtaining supergain are investigated. The admittance constraints are formulated as linear constraints for specific cases as well as more general, quadratic constraints, which lead to the solution of an equivalent convex relaxation formulation. A design example for an electrically small superdirective rectenna is provided where an upper bound for the rectifier efficiency is computed.
\end{abstract}

Index Terms-Supergain, superdirectivity, electrically small antenna, rectenna, rectifier, energy harvesting, wireless power transmission, convex optimization, semidefinite relaxation, robust optimization.

\section{INTRODUCTION}

C ONVEX optimization provides for a computationally efficient numerical method to obtain globally optimum solutions of certain types of optimization problems exploring the property of convexity [1]. A challenge then becomes that of being able to formulate an optimization problem in a desired standard convex optimization form that can be efficiently solved. Convex optimization techniques have been applied in antenna problems and specifically in antenna pattern synthesis, optimizing the current distribution of electically small antennas, investigating superdirectivity and optimizing antenna array gain [2]-[5].

Electrically small antennas have been defined by Wheeler [6] as ones that their largest dimension measured in wavelengths $\alpha / \lambda$ is smaller than the radian-length $(2 \pi)^{-1} \approx 0.159$. This is alternatively expressed as $k \alpha<1$, where $k=2 \pi / \lambda$ is the wavenumber. Smaller limits have also been proposed in the literature, however, similarly to [7], we adopt this limit in this work due to the fact that we consider arrays of antennas. Due to their electrically small dimensions they present an input impedance which has a small real part corresponding to the radiation resistance and a large reactive part. Naturally, they find good application in miniature wireless devices or in low frequency systems.

A. Georgiadis e-mail: apostolos.georgiadis@ieee.org.

Nuno Borges Carvalho email: nbcarvalho@ua.pt.

Manuscript received June 7, 2021; revised .
Superdirectivity is defined as the property of antennas achieving very high directivity despite a small electrical size. Uzkov [8] showed that the directivity of linear arrays of $N$ isotropic elements approaches $N^{2}$ as the element spacing tends to zero, while it is equal to $N$ when the inter-element distance is a multiple of $d=\lambda / 2$. The latter can be achieved by antenna arrays with broadside radiation patterns where their elements are excited uniformly with equal amplitude and phase and is known as nominal directivity [9]. Endfire antenna arrays are capable of achieving a directivity beyond the nominal value $N$ and towards the $N^{2}$ super-directivity limit as the element spacing tends to small values $\leq 0.2 \lambda$.

Superdirective arrays of electrically small antennas have been considered in the literature in order to maximize the directivity and gain of the antenna system while maintaining an electrically small size [7]. Furthermore, arrays of parasitically loaded elements have been implemented [7], inspired by earlier works on loaded scatterers [10] and control of directivity using reactively loaded parasitic elements [11]. A challenge with implementing such arrays is that the conditions for super-directivity are very sensitive to variations in the array design parameters such as the element excitations. In order to reduce sensitivity, one can design an array with a higher directivity than the nominal value but below the maximum obtainable directivity value [12].

Electrically small rectennas have also been investigated in the literature [13]-[15] with direct application in RF energy harvesting and wireless powering for miniature wireless sensors [16]. In this work, we explore the possibility of designing superdirective electrically small rectennas, which are suitable in applications where preferred directions of incoming RF power are available. Some preliminary considerations have been presented in [17] without the convex optimization formulation.

Antenna arrays with closely spaced reactively loaded elements and high directivity have been successfully demonstrated in the literature, using however global optimization techniques [18]. Furthermore, convex optimization formulations for electrically small antennas have been presented in the literature, where the objective function is either to minimize the radiation (and ohmic) losses of the antenna resulting in the maximization of its directivity (gain), or to minimize the reactive power resulting in the maximization of the gainquality factor $G / Q$ ratio [3], [4], [19]. 
In this work, a convex optimization formulation for arrays of reactively loaded dipole antennas is presented. Inspired by [10], [11] and [3], [4], [19] an optimization problem is defined for arrays of reactively loaded elements but the formulation is based on excitation voltages, minimizing the dimension of the unknowns to the number of input ports. Furthermore, different expressions for constraints on the input impedance are proposed which allow for an easy computation of the reactive load values. In addition, the sensitivity - directivity trade-off is investigated. Finally, a rectenna design example is presented using a single diode shunt rectifier impedance matched to the antenna, providing a maximum efficiency limit. The optimization results are obtained using freely available electromagnetic simulation and optimization software thus allowing for a flexible and easy implementation.

\section{CONVEX OPTIMIZATION FORMULATION}

The standard form of a convex optimization problem is [1]:

$$
\begin{array}{rlrl}
\text { minimize } & f_{0}(x) & & \\
\text { subject to } & f_{i}(x) \leq 0 & i=1, \ldots, m \\
& h_{i}(x)=a_{i}^{T} x-b_{i}=0 & i=1, \ldots, p
\end{array}
$$

where the objective $f_{0}$ and the inequality functions $f_{i}(x)$ are convex functions and the equality constraints $h_{i}(x)$ are affine (ie comprise a linear term plus a constant) [1].

\section{A. Arrays of fed elements}

An array of $N$ electrically small identical dipoles is considered. For simplicity we consider $f=300 \mathrm{MHz}$ which corresponds to a free space wavelength of $\lambda_{0}=1 \mathrm{~m}$. Furthermore, wire dipoles with radius $r=0.001 \mathrm{~m}$ and length $L=0.1 \mathrm{~m}$ are considered arranged along the $y$ axis with spacing $d$. Copper wire is assumed with conductivity $\sigma=5.6 \cdot 10^{7} \mathrm{~S} / \mathrm{m}$.
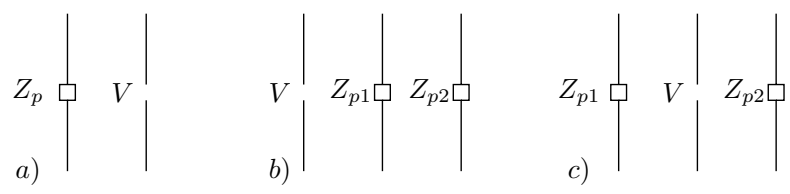

Fig. 1. Electrically small superdirective antenna arrays with one fed dipole and additional reactively loaded dipole elements, a) $N=2$ with one reflector, b) $N=3$ with two directors and c) $N=3$ with one director and one reflector. The dipoles are assumed to be directed along the $\mathrm{z}$-axis and the arrays along the $\mathrm{y}$-axis.

The dipoles have been simulated with the moment method code by Richmond [20], [21], implemented in house in Octave [22] and the results have been verified with NEC [23], [24]. The electromagnetic analysis computes the complex admittance (or impedance) matrix $Y$ providing a linear matrix equation relating the complex current $I$ and the complex excitation voltage $V$ at the input ports of the dipoles,

$$
I=Y V
$$

where the current $I$ and the voltage $V$ vectors have a dimension $N$ equal to the number of excitation ports (which is equal to the number of dipoles in the cases considered) and $Y$ is a diagonally symmetric square complex matrix with dimension $N \times N$. The complex impedance can be separated into the conductance and reactance matrices as $Y=G+j B$. The conductance matrix $G$ is a symmetric matrix, ie $G^{T}=G$ where the superscript ()$^{T}$ denotes the transpose operation. In the following, complex vectors $x$ and matrices $A$ are converted into real vectors $x_{R}$ and real matrices $A_{R}$ with double dimensions, using the following convention:

$$
\begin{array}{r}
x \rightarrow x_{R}=\left[\begin{array}{l}
\Re x \\
\Im x
\end{array}\right] \\
A \rightarrow A_{R}=\left[\begin{array}{cc}
\Re A & -\Im A \\
\Im A & \Re A
\end{array}\right]
\end{array}
$$

where $\Re$ and $\Im$ denote the real and imaginary parts respectively. For example, the admittance matrix is expressed as

$$
Y_{R}=\left[\begin{array}{cc}
G & -B \\
B & G
\end{array}\right]=G_{R}+B_{R}
$$

with

$$
\begin{gathered}
G_{R}=\left[\begin{array}{cc}
G & 0 \\
0 & G
\end{array}\right] \\
B_{R}=\left[\begin{array}{cc}
0 & -B \\
B & 0
\end{array}\right]
\end{gathered}
$$

The array directivity for a radiation peak along a direction $\theta_{0}=\phi_{0}=90^{\circ}$ (assuming the dipoles are directed along the $\mathrm{z}$-axis and the arrays along the $\mathrm{y}$-axis) is given as

$$
D_{0}=\frac{4 \pi}{\eta} \frac{V_{R}^{T} F_{R 0}^{T} F_{R 0} V_{R}}{V_{R}^{T} G_{R} V_{R}}
$$

where $\eta=120 \pi$ and the radiated electric field amplitude is $E_{\theta}\left(\theta_{0}, \phi_{0}\right)=F_{0} V \Rightarrow E_{R \theta}\left(\theta_{0}, \phi_{0}\right)=F_{R 0} V_{R}$. The optimization problem is formulated by imposing a fixed value on the radiated field amplitude $E_{\theta}\left(\theta_{0}, \phi_{0}\right)$ and minimizing the radiated power $\left(P_{r}=V_{R}^{T} G_{R} V_{R}\right)$, expressed in standard form as

$$
\begin{aligned}
\text { minimize } & r \\
\text { subject to } & V_{R}^{T} G_{R} V_{R} \leq r \\
& \left(F_{R 0} V_{R}\right)(2)=-1
\end{aligned}
$$

The first nonequality constraint is a quadratic constraint on the excitation voltages, known as a convex cone constraint [1]. The second constraint is an equality constraint imposing the value -1 to the imaginary part of the electric field amplitude $E_{R \theta}$ selected by the index (2), similarly to [25]. Actually, any fixed value for the real or imaginary part of the field suffices, since the objective of the constraint is to limit the numerator of the directivity to a fixed value.

The solution of the convex optimization problem (9) provides the array excitations corresponding to the maximum directivity. If the admittance matrix $Y_{R}$ and by extension the conductance matrix $G_{R}$, includes ohmic losses due to the finite conductivity of the wires, then the optimization problem provides the maximum gain instead of the maximum directivity. The formulation of problem (9) has been proposed in the literature [3], [4] however in terms of the current distribution in the dipoles rather than the excitation voltages. 


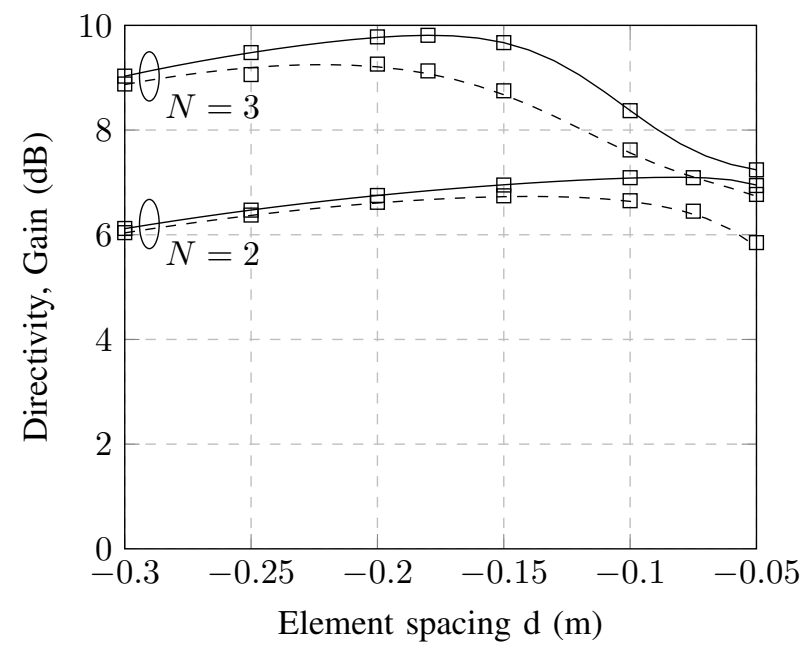

Fig. 2. Optimum directivity (solid line) and gain (dashed line) versus interelement distance for dipole arrays of $N=2$ and $N=3$ elements, obtained using (9). Results obtained with NEC indicated with square marks.

The single element has a simulated directivity $D=1.77 \mathrm{~dB}$ and gain $G=1.72 \mathrm{~dB}$. The maximum broadside (nominal) directivity of a uniformly excited array is therefore $D_{b}=$ $4.78 \mathrm{~dB}$ and $D_{b}=6.54 \mathrm{~dB}$ for $N=2$ and $N=3$ dipole elements respectively. Higher values than these correspond to superdirective arrays, with a theoretically maximum limit of $D_{s}=7.79 \mathrm{~dB}$ and $D_{s}=11.31 \mathrm{~dB}$ for $N=2$ and $N=3$ dipole elements respectively.

The optimization problems in this work have been solved in Octave using the YALMIP [26] toolbox with the convex optimization solver SeDuMi [27].

The maximum endfire directivity and gain for a two element and a three element array obtained by (9) are shown in Fig. 2. The results have been verified by applying the obtained complex excitation voltages from (9) into NEC which resulted in the values indicated by the square marks in the plot, showing a very good agreement with the results obtained using our in house implemented Richmond moment method code. Using the Richmond code, 4 equal segments per dipole were defined which correspond to 3 overlapping current modes, each spanning over two consequtive segments. In contrast, NEC defines 3 segments per dipole and 1 mode per segment. Nonetheless, the directivity and gain values have been remarkably close, as shown in Fig. 2.

In Fig. 2 one can see that for two dipoles a maximum directivity of $7.1 \mathrm{~dB}$ is obtained at approximately $0.08 \mathrm{~m}$ interelement distance, however the gain takes its maximum value of $6.75 \mathrm{~dB}$ at approximately $0.14 \mathrm{~m}$ distance. In the case of three dipoles the maximum directivity of $9.8 \mathrm{~dB}$ is obtained at $0.18 \mathrm{~m}$ inter-element distance, while a maximum gain of $9.25 \mathrm{~dB}$ is obtained at $0.22 \mathrm{~m}$ distance.

\section{B. Constraints for loaded elements}

The input admittance $Y_{i}$ at an excitation point $i$ is an active admittance given by

$$
Y_{i n, i}=\frac{1}{V_{i}} \sum_{j=1}^{N} Y_{i j} V_{j}
$$

where the index $j$ spans over the $N$ excitation points. In order to impose a constraint over the real part $\alpha$ or the imaginary part $\beta$ of the input admittance $y=\alpha+j \beta$ of an excitation point $i$ one needs to separate the real and imaginary parts of (10), resulting in

$$
\begin{aligned}
& \sum_{j=1}^{N} \Re\left\{V_{i}^{*} Y_{i j} V_{j}\right\}=\alpha\left|V_{i}\right|^{2} \\
& \sum_{j=1}^{N} \Im\left\{V_{i}^{*} Y_{i j} V_{j}\right\}=\beta\left|V_{i}\right|^{2}
\end{aligned}
$$

The above equations can be expressed after some algebra as quadratic matrix inequalities of the excitation vector $V_{R}$. One may therefore write

$$
\begin{gathered}
V_{R}^{T} Y_{R i \alpha} V_{R}=0 \\
V_{R}^{T} Y_{I i \beta} V_{R}=0
\end{gathered}
$$

The real matrices $Y_{\text {Ri } \alpha}$ and $Y_{I i \beta}$ are computed by expanding (11) and (12) respectively. It turns out that $Y_{R i \alpha}$ can be constructed by taking a $2 N \times 2 N$ zero matrix and setting its $i$ line and $i+N$ column to be equal to line $i$ and line $i+N$ respectively of $\left(Y_{R}-\alpha I_{2 N}\right)$ and then setting the common element between the $i$ line and $i+N$ column

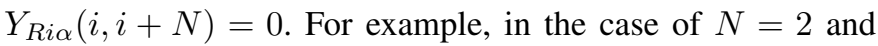
for element $i=2$, one has

$$
Y_{R 2 \alpha}=\left[\begin{array}{cccc}
0 & 0 & 0 & B_{R 12} \\
G_{R 21} & G_{R 22}-\alpha & -B_{R 21} & 0 \\
0 & 0 & 0 & G_{R 12} \\
0 & 0 & 0 & G_{R 22}-\alpha
\end{array}\right]
$$

One way to introduce a reactive loading element into the optimization problem (9) is to impose a constraint that the input admittance at excitation point $i$ is imaginary, ie to include a constraint of the form (13) with $\alpha=0$. Therefore the problem becomes

$$
\begin{array}{rlr}
\text { minimize } & r & \\
\text { subject to } & V_{R}^{T} G_{R} V_{R} & \leq r \\
& \left(F_{R 0} V_{R}\right)(2) & =-1 \\
V_{R}^{T} Y_{R i 0} V_{R} & =0 \quad \forall i=1, \ldots
\end{array}
$$

where the index $i$ runs over all the reactive loads in the array. Once the optimum excitation voltages are obtained, one computes the corresponding reactive loading element $B_{L i}$ as

$$
B_{L i}=-B_{i n, i}
$$

A corresponding inductance or capacitance can be easily computed from the imaginary reactive load $Y_{L i}=j B_{L i}$.

However, problem (16) is not a convex optimization problem because of the quadratic equality constraint for the loading 


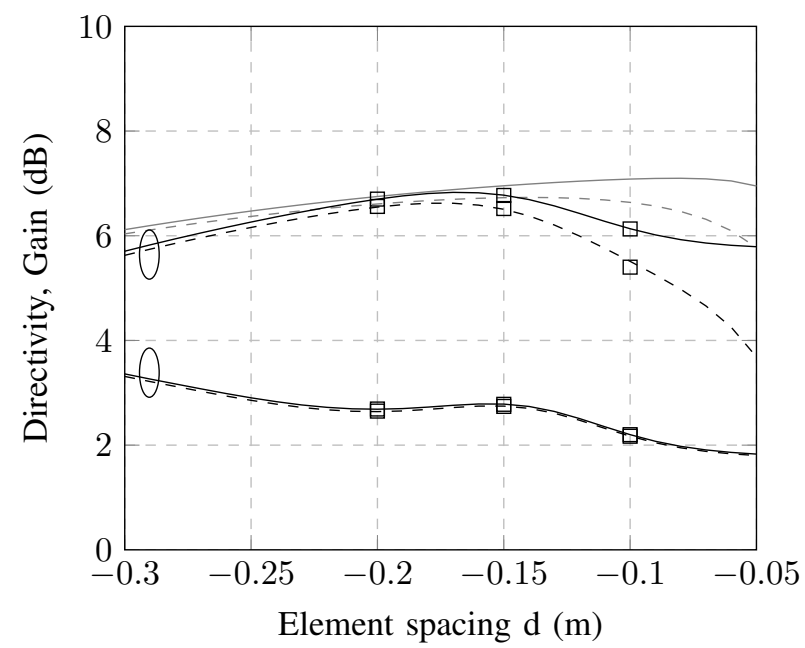

Fig. 3. Directivity (solid line) and gain (dashed line) solutions of a $N=2$ element array comprising one fed element and a reactively loaded reflector element obtained using (20). The directivity and gain values corresponding to the same set of admittance constraints are indicated by circles. NEC results are shown with square marks. The solutions corresponding to all elements being excited from Fig. 2 are shown in gray.

elements. One way to convert the problem into a convex optimization problem would be to substitute the quadratic equalities with inequalities of the form $-e \leq V^{T} Y_{R i}(0) V \leq e$ with $e$ being a very small number e.g. $10^{-12}$. This provides an approximate solution.

A second way to convert problem (16) into a convex optimization problem is to explore the structure of the constraint (13) or equivalently (11). It is easy to verify that it is possible to substitute the quadratic constraint (11) by a pair of affine constraints as

$$
\begin{gathered}
\Im\left\{V_{i}\right\}=0 \\
\sum_{j=1}^{N} \Re\left\{Y_{i j} V_{j}\right\}=\alpha \Re\left\{V_{i}\right\}
\end{gathered}
$$

or

$$
\begin{gathered}
\Re\left\{V_{i}\right\}=0 \\
\sum_{j=1}^{N} \Im\left\{Y_{i j} V_{j}\right\}=\alpha \Im\left\{V_{i}\right\}
\end{gathered}
$$

The convex optimization problem is thus formulated as

$$
\begin{array}{cc}
\text { minimize } & r \\
\text { subject to } & V_{R}^{T} G_{R} V_{R} \leq r \\
\left(F_{R 0} V_{R}\right)(2) & =-1 \\
V_{R}(i+N)=0 & \text { and } c_{R 1} V_{R}=0 \\
& \text { or } \\
V_{R}(i)=0 & \text { and } c_{R 2}^{T} V_{R}=0 \\
\forall i & =1, \ldots
\end{array}
$$

where $c_{R 1}$ is the $i$ row of $Y_{R i 0}$ and $c_{R 2}$ is the $i+N$ column of $Y_{R i 0}$.

In the case of $N=2$ dipoles, a topology of one fed element and one parasitic reflector element with a reactive load is selected, as shown in Fig. 1a. In order to impose a constraint for an imaginary input impedance at the terminals of the reflector dipole element one has two possibilities corresponding to selecting the real part or the imaginary part of the excitation voltage to be equal to zero in (20). The optimization results are shown in Fig. 3, where the case for both dipoles being excited is included for comparison. One can see that one solution leads to a low directivity and gain and is thus not useful, but the other solution leads to high directivity values which are approximately equal to the solution corresponding to both elements being excited for element spacings in the range $0.15 \mathrm{~m}$ to $0.2 \mathrm{~m}$.

The results have been verified using NEC. There was a difference in the port admittance values of the dipoles between the two electromagnetic simulation codes, which is expected to be minimized by increasing the number of segments per dipole. However, the convergence of the electromagnetic simulation codes is not the object of this work and consequently the number of segments per dipole was kept relatively small in order to minimize the simulation time. In order to overcome the difference in the obtained admittance values, in a first step we apply the excitation voltages for both elements obtained from (20) in NEC. As expected, these result in an imaginary input admittance for the parasitic dipole. We then compute the reactive load value by taking the negative (conjugate) of the imaginary input admittance provided by NEC. In a second step, we apply a unit excitation voltage to the fed element and the computed reactive load to the parasitic element and obtain the desired directivity and gain values indicated using square marks in the figure, showing an exceptional agreement with the results obtained with the Richmond code.

The case of $N=3$ dipole elements with one fed element and two reactively loaded directors (Fig. 1b) is shown in Fig. 4. Furthermore, the case of $N=3$ dipole elements with one fed element, one reactively loaded reflector and one reactively loaded director (Fig. 1c) is shown in Fig. 5. In each configuration there are four possible combinations in (20) which lead to a corresponding solution branch. One can see that in each case there exists one solution branch which results in a good directivity and gain value for an inter-element distance around $0.2 \mathrm{~m}$, however all solutions are more than 1 $\mathrm{dB}$ below the values obtained for the case where all dipoles are excited. This result hints towards the fact that the admittance constraint results in a sub-optimum solution.

\section{Semidefinite Relaxation}

An alternative way to implement a convex optimization formulation with a constraint on an active admittance value without imposing any constraint on the excitation voltage of the corresponding port, is to apply a semidefinite relaxation [28]. In a first step, one takes advantage of the property of the trace $\operatorname{Tr}()$ of a matrix $V_{R}^{T} Y_{R i 0} V_{R}=$ $\operatorname{Tr}\left(V_{R}^{T} Y_{R i 0} V_{R}\right)=\operatorname{Tr}\left(Y_{R i 0} V_{R} V_{R}^{T}\right)$, where the first equality holds because $V_{R}^{T} Y_{R i 0} V_{R}$ is a scalar. Letting $X=V_{R} V_{R}^{T}$, where $X$ is a square symmetric matrix $\left(X^{T}=X\right)$, one has $V_{R}^{T} Y_{R i 0} V_{R}=\operatorname{Tr}\left(Y_{R i 0} X\right)$. This way the original quadratic equality constraint in the vector $V_{R}$ is substituted by a linear 


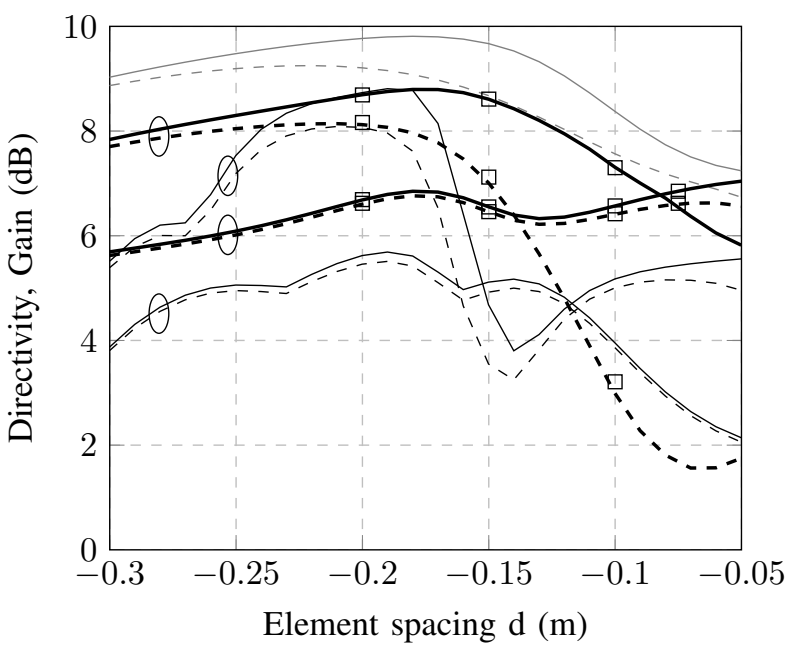

Fig. 4. Directivity (solid line) and gain (dashed line) solutions of a $N=3$ element array comprising one fed element and two reactively loaded director elements obtained using (20). The directivity and gain values corresponding to the same set of admittance constraints are indicated by circles. NEC results are shown with square marks. The solutions corresponding to all elements being excited from Fig. 2 are shown in gray.

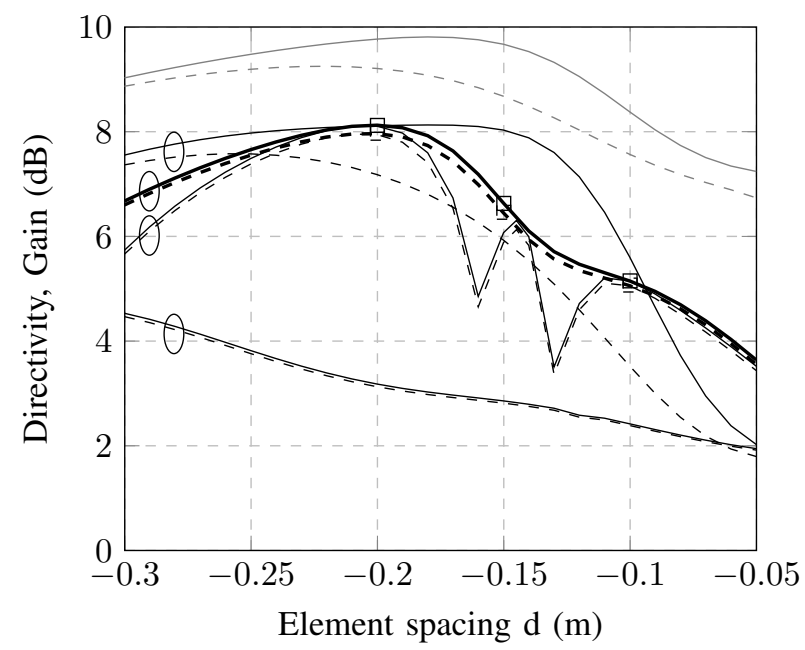

Fig. 5. Directivity (solid line) and gain (dashed line) solutions of a $N=3$ element array comprising one fed element, one a reactively loaded reflector and one reactively loaded director obtained using (20). The directivity and gain values corresponding to the same set of admittance constraints are indicated by circles. NEC results are shown with square marks. The solutions corresponding to all elements being excited from Fig. 2 are shown in gray.

constraint in the square symmetric matrix $X$, at the expense of increasing the number of optimization variables, since the original vector $V_{R}$ has $2 N$ variables whereas the square matrix $X$ has $2 N(2 N+1) / 2$ variables. The optimization problems are equivalent under the constraint $X=V_{R} V_{R}^{T}$ which is not a convex constraint. In a second step, one relaxes the above (nonconvex) equality into a convex inequality $X \geq V_{R} V_{R}^{T}$. The inequality is then expressed in the form of an equivalent (convex) matrix inequality [28]

$$
X \geq V_{R} V_{R}^{T} \Leftrightarrow\left[\begin{array}{cc}
X & V_{R} \\
V_{R}^{T} & 1
\end{array}\right] \succeq 0
$$

which can be directly set in standard convex optimization toolboxes, such as the SeDuMi used in this work. The symbol $\succeq$ defines a matrix inequality where corresponding matrix is positive semidefinite $\left(A \succeq 0 \Leftrightarrow x^{T} A x \geq 0, \forall x\right)$. It should be noted that in [19], semidefinite relaxation was used to substitute quadratic equality constraints with respect to the quadratic forms for the antenna current distribution corresponding to the antenna radiated and stored power.

The relaxed constraint leads to a lower bound of the optimization problem, the two problems being equivalent if the rank of matrix $X$ is equal to one [28]. The rank constraint is however also a nonconvex constraint and thus not useful in our convex optimization formulation. In order to mitigate the effect of the relaxation we add an optimization objective of minimizing the trace of matrix $X$ in order to minimize the rank of matrix $X$. The rank of the square matrix $X$ is equal to the number its non-zero eigenvalues, while the trace of matrix $X$ is equal to the sum of its eigenvalues. It has been shown however, that minimizing the trace leads to a low rank matrix [29]. The use of a heuristic method such as this one in order to minimize the rank of a matrix was also suggested in [5].

The convex optimization formulation becomes

$$
\begin{aligned}
\text { minimize } & r+t \cdot r_{1} \\
\text { subject to } \quad-F_{R 0} V_{R}(2) & =-1 \\
\operatorname{Tr}\left(G_{R} X\right) & \leq r \\
\operatorname{Tr}\left(Y_{R i 0} X\right) & =0 \\
{\left[\begin{array}{cc}
X & V_{R} \\
V_{R}^{T} & 1
\end{array}\right] } & \succeq 0 \\
\operatorname{Tr}(X) & \leq r_{1}
\end{aligned}
$$$$
\operatorname{Tr}\left(Y_{R i 0} X\right)=0 \quad \forall i=1, \ldots
$$

The scalar objective $r+t \cdot r_{1}$ of the optimization problem combines two different objectives corresponding to $r$ and $r_{1}$ respectively with a weighting parameter $t$. The two objectives correspond to the minimization of the radiated power through $r$ and the minimization of the trace of $X$ through $r_{1}$. The formulation of the scalar objective allows us to trace through the pairs of optimum values for the two objectives corresponding to each weight value defining what is known as the Pareto front of the vector optimization problem defined by the two objectives (22) [1].

The solution of (22) for $N=2$ is shown in Fig. 6 where the result is almost identical with the better one of the two solutions of (20) shown in Fig. 3. The solution of (22) for $N=3$ is shown in Fig. 7. In this case, there is a substantial improvement over the results obtained with (20), shown in Fig. 4 and Fig. 5. One can see that the solution corresponding to one reflector and one director element reaches the limit corresponding to three excited elements for spacing values in the range $0.25 \mathrm{~m}$ to $0.19 \mathrm{~m}$ approximately.

The solution corresponding to two director elements also reaches a maximum directivity value close to the limiting value of three excited elements albeit for a small range of element spacing values near $0.17 \mathrm{~m}$. It should be noted that solving (22) we frequently encountered numerical convergence errors which did not always allow to obtain an optimum solution. 


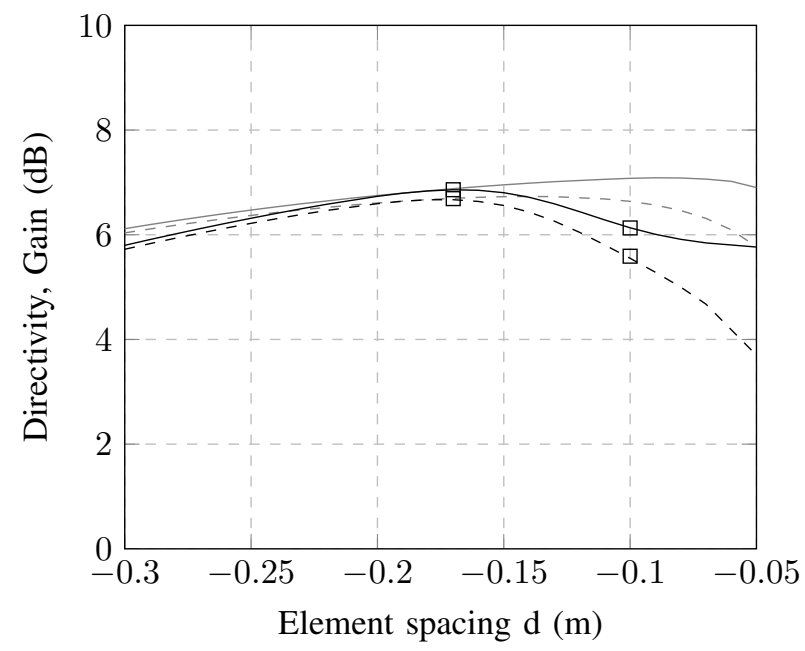

Fig. 6. Optimum directivity and gain of a $N=2$ element array with one fed dipole and one reactively loaded reflector dipole computed with the semidefinite relaxation formulation (22). The optimization results corresponding to both elements being excited obtained with (22) are shown in gray. NEC results are indicated with square marks.

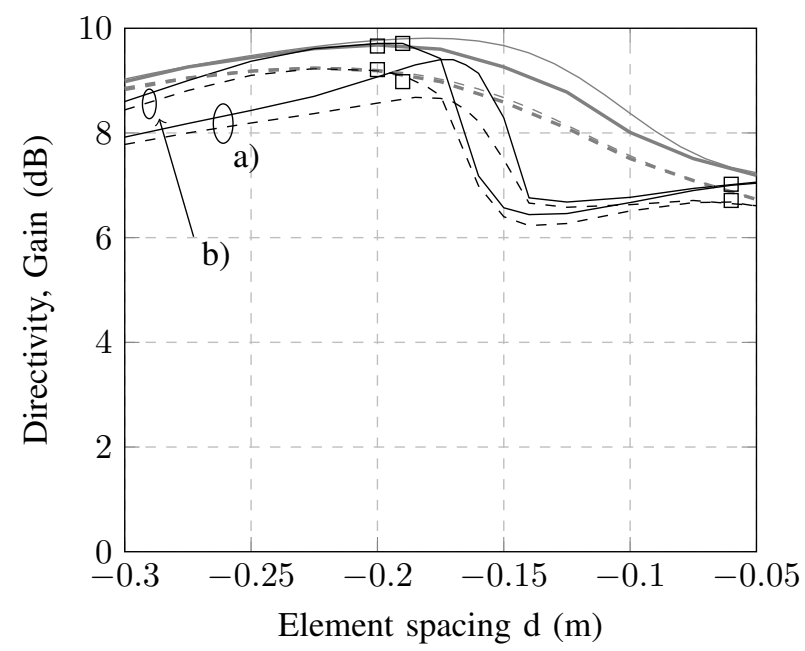

Fig. 7. Optimum directivity and gain of a $N=3$ element array with one fed dipole and a) two reactively loaded director dipoles and b) one reactively loaded reflector dipole and one director dipole, computed with the semidefinite relaxation formulation (22). The optimization results corresponding to all three elements being excited obtained with (20) and (22) are shown in gray and thick gray respectively. NEC results are indicated with square marks.

In order to investigate this further, we traced the solutions for three excited elements using (22) by deleting the equality constraint corresponding to the purely imaginary admittance and encountered the same numerical problems which allowed us to obtain a limiting solution curve slightly below the one obtained with (20). Further investigation is necessary, however it is likely that the numerical problems are due to the small admittance values which maybe mitigated by properly scaling the objective and constraints.

\section{SENSITIVITY}

The solutions corresponding to maximum directivity are typically very sensitive to parameter variations [12], such as the excitation voltages or the values of the reactive loads. In [12], a sensitivity parameter was defined in order to obtain a measure of the variation of the directivity due to variations in the antenna array excitations and it was suggested that one may sacrifice some directivity in order to design an antenna array that is easy (or even practically feasible) to implement. In terms of the optimization discipline the formulation corresponds to a robust optimization problem [1]. In fact one may distinguish between variations in the array pattern or variations in the array excitations and accordingly define a robust optimization problem [30]. In [3] a convex optimization formulation was proposed that was directed at optimizing the current distribution of an antenna in order to maximize the gain over quality factor $(\mathrm{G} / \mathrm{Q})$ ratio for a minimum directivity value $D_{0}$ of an antenna. This was done by defining an objective function that mimimizes the reactive energy of the antenna, where the electric and magnetic energy of an antenna were computed as quadratic forms of the current [3]. In [19] these optimization problems were solves applying a semidefinite relaxation formumation. Inspired by the works of [3], [12], [19], we present a corresponding semidefinite relaxation formulation of a convex optimization problem with an objective to minimize the reactive energy, while imposing the necessary constraints on the input admittance of the parasitic elements as well as a constraint on a minimum directivity.

The stored electric $W_{e}$ and magnetic $W_{m}$ energies are approximated as [25]

$$
\begin{aligned}
W_{e} & \approx \frac{1}{8} I^{H}\left(\frac{\partial X}{\partial \omega}-\frac{X}{\omega}\right) I=\frac{1}{4 \omega} I^{H} X_{e} I \\
W_{m} & \approx \frac{1}{8} I^{H}\left(\frac{\partial X}{\partial \omega}+\frac{X}{\omega}\right) I=\frac{1}{4 \omega} I^{H} X_{m} I
\end{aligned}
$$

where $Z=R+j X$ the antenna impedance martrix. Equivalently, in terms of the admittance matrix $Y=G+j B$, the above expressions are computed as

$$
\begin{gathered}
W_{e} \approx \frac{-1}{8} V^{H}\left(\frac{\partial B}{\partial \omega}-\frac{B}{\omega}\right) V=\frac{1}{4 \omega} V^{H} B_{e} V \\
W_{m} \approx \frac{-1}{8} V^{H}\left(\frac{\partial B}{\partial \omega}+\frac{B}{\omega}\right) V=\frac{1}{4 \omega} V^{H} B_{m} V
\end{gathered}
$$

where the derivatives of the susceptance are computed numerically using finite differences. The antenna quality factor $\mathrm{Q}$ is then computed as

$$
Q \approx \frac{\max \left\{V^{H} B_{e} V, V^{H} B_{m} V\right\}}{V^{H} G V}
$$




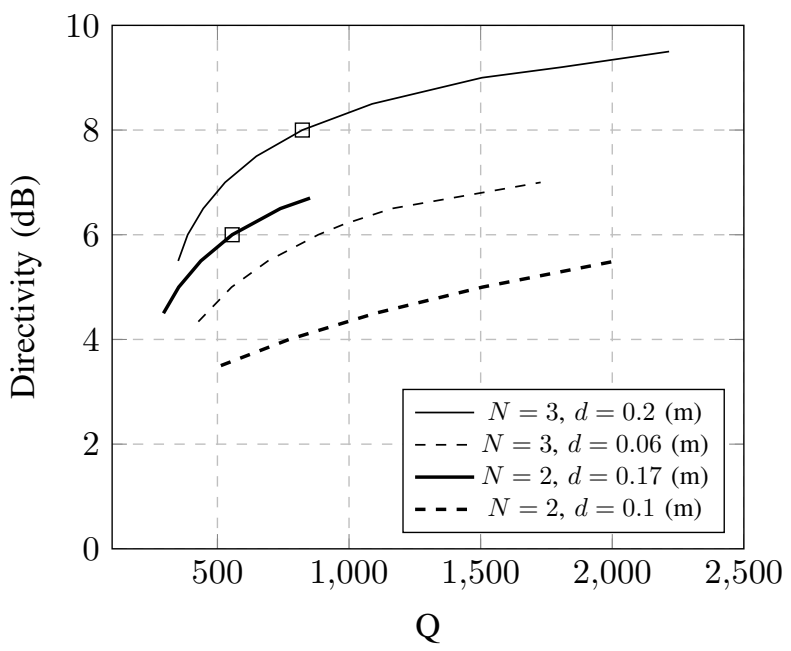

Fig. 8. Directivity vs quality factor $\mathrm{Q}$ trade-off, computed for selected antenna configurations using formulation (28). NEC results are indicated with square marks.

The convex optimization problem is formulated as

$$
\begin{aligned}
\text { minimize } & r+t \cdot r_{1} \\
\text { subject to } \quad-F_{R 0} V_{R}(2) & =-1 \\
\operatorname{Tr}\left(B_{e R} X\right) & \leq r \\
\operatorname{Tr}\left(B_{m R} X\right) & \leq r \\
\operatorname{Tr}\left(G_{R} X\right) & \leq \frac{4 \pi}{\eta D_{0}} \quad \forall i=1, \ldots \\
\operatorname{Tr}\left(Y_{R i 0} X\right) & =0 \\
{\left[\begin{array}{cc}
X & V_{R} \\
V_{R}^{T} & 1
\end{array}\right] } & \succeq 0 \\
\operatorname{Tr}(X) & \leq r_{1}
\end{aligned}
$$

where the constraints on the stored electric and magnetic energy have been formulated using the corresponding real matrices $B_{e R}$ and $B_{m R}$ according to (4) and the radiated power constraint on $G_{R}$ is used to impose a minimum directivity $D_{0}$ as in [3], [19]. In Fig. 8 formulation (28) has been used to compute trade-offs between directivity and quality factor for selected antenna spacings.

\section{RECTIFIER}

Once an suitable antenna configuration is selected based on a desired directivity/gain value, a rectifier circuit can be designed in order to implement a rectenna as an RF energy harvester with optimum efficiency. Due to the fact that rectifier circuits have an input impedance with a large reactive part one may try to take advantage of the complex input impedance of electrically small antennas in order to implement a conjugate impedance match for maximum power transfer. As an example, we select an antenna comprising $N=2$ elements, one fed element and one reflector with a distance of $0.17 \mathrm{~m}$ having a directivity of $6 \mathrm{~dB}$, based on Fig. 8. This configuration results in an input antenna impedance $Z_{A}=2.2-j 1068$.

A shunt diode rectifier circuit was selected with a series inductor $L_{1}$ to a parallel RC load at its output in order to filter the dc voltage, shown in Fig 9. Using this circuit topology, it is possible to select the inductance value in order to provide a desirable input resistance, as shown in Fig. 10. The simulation results of Fig. 10 were obtained using QucsStudio [31]. An SMS7630 diode was selected and a nonlinear model using a parasitic inductance $L_{p}=0.6 \mathrm{nH}$ and parasitic capacitance $C_{p}=0.25 \mathrm{pF}$ were used, as in [32], [33]. In order to obtain the results of Fig. 10, the input source impedance and output load of the rectifier were optimized in QucsStudio for each value of $L_{1}$ and for a specific avalable input power in order to maximize the RF-dc conversion efficiency. A value of $L_{1}=60 \mathrm{nH}$ was selected in order to provide the desired input resistance of $2.2 \Omega$ for an available input power of $-20 \mathrm{dBm}$. Then, a series inductor $L_{2}$ is placed between the rectifier and the antenna in order to provide the desired conjugate match for the reactive part of the antenna impedance. The simulated performance of the rectifier versus frequency for an input available power of $-20 \mathrm{dBm}$ is plot in Fig. 11, showing a maximum efficiency of approximately $61 \%$ for an output load of $R_{L}=12.4 \mathrm{~K} \Omega$.

This value represents an upper bound on the achievable efficiency using the selected diode and for the given input power at $300 \mathrm{MHz}$. Ideal inductor models were used in the simulations. The obtained RF-dc efficiency will be reduced once realistic inductor models as well as the rectifier circuit layout are considered.

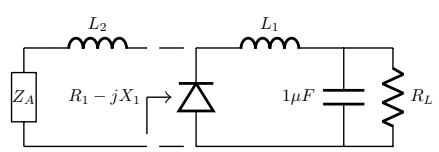

Fig. 9. Rectenna circuit schematic.

\section{DISCUSSION}

These results demonstrate a systematic approach for the design of superdirective electrically small antennas and rectennas. There are several additional challenges that originate from this work, such as increasing the real part of the antenna input impedance for example by using folded fed elements as indicated in [7], addressing in more detail the sensitivity and implementing a prototype.

One should emphasize however, the fact that the provided optimization formulation are very easy to implement. Specifically the provided convex optimization formulations require the admittance matrix corresponding to the ports of the antenna system, which can be directly obtained by any commercial electromagnetic simulation software. They also require the radiated field at one desired direction that corresponds to the maximum directivity of the antenna system and this is can also be obtained by any antenna electromagnetic simulation package. Specifically, as described in [10], the radiated field is obtained as a superposition of the radiated fields obtained by exciting each port of the antenna system by a unit voltage with all other ports shorted.

\section{CONCLUSION}

A convex optimization formulation was presented for electrically small antennas and rectennas based on arrays of 


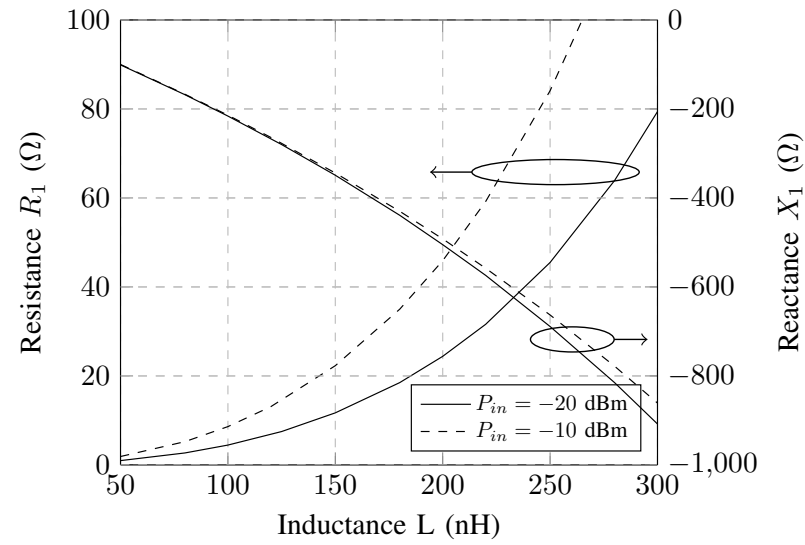

Fig. 10. Rectifier optimum source impedance.

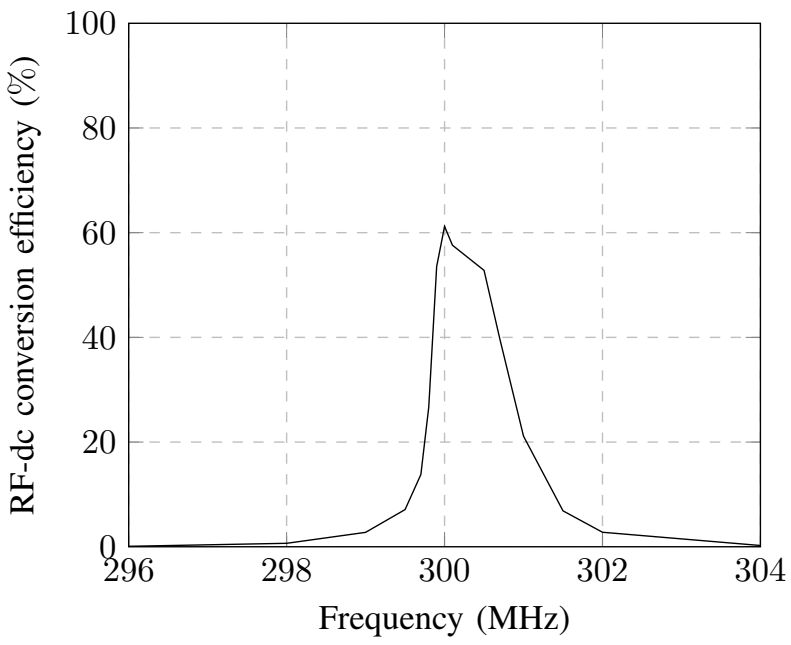

Fig. 11. Theoretical maximum efficiency versus frequency, $L_{1}=60 \mathrm{nH}$, $L_{2}=501.8 \mathrm{nH}, R_{L}=12.4 \mathrm{k} \Omega, P_{A}=-20 \mathrm{dBm}$.

radiating elements comprising a fed element and reactively loaded parasitic elements. Different formulations were provided implementing constraints on the input admittance of the radiating elements in addition to minimizing the antenna $\mathrm{Q}$ for a specific directivity. The formulation was based on the input voltage excitations of the radiating elements resulting in optimization problems with a small number of unknowns that can be solved efficiently using freely or commercially available electromagnetic simulation and optimization packages.

\section{REFERENCES}

[1] S. Boyd and L. Vandenberghe, Convex Optimization. Cambridge University Press, 2004.

[2] H. Lebret and S. Boyd, "Antenna array pattern synthesis via convex optimization," IEEE Transactions on Signal Processing, vol. 45, no. 3, pp. 526-532, March 1997.

[3] M. Gustafsson and S. Nordebo, "Optimal antenna currents for q, superdirectivity and radiation patterns using convex optimization," IEEE Transactions on Antennas and Propagation, vol. 61, no. 3, pp. 11091118, March 2013.

[4] L. Jelinek and M. Capek, "Optimal currents on arbitrary shaped surfaces," IEEE Transactions on Antennas and Propagation, vol. 65, no. 1, pp. 329-341, January 2017.
[5] J. Lavaei, A. Babakhani, A. Hajimiri, and J. C. Doyle, "Solving largescale linear circuit problems via convex optimization," in Proc. 48th IEEE Conference on Decision and Control (CDC), 2009, pp. 49774984.

[6] H. A. Wheeler, "Fundamental limitations of small antennas," Proceedings of the IRE, vol. 35, no. 12, pp. 1479-1484, December 1947.

[7] A. D. Yaghjian, T. H. O'Donnell, E. Altshuler, and S. R. Best, "Electrically small supergain end-fire arrays," Radio Science, vol. 43, no. RS3002, pp. 1-13, 2008.

[8] A. Uzkov, "An approach to the problem of optimum directive antenna design," Comptes Rendus (Doklady) de l' Academie de Sciences de l' URSS, vol. III, p. 35, 1946.

[9] C. Tai, "The optimum directivity of uniformly spaced broadside arrays of dipoles," IEEE Transactions on Antennas and Propagation, vol. 12, no. 4, pp. 447-454, July 1964.

[10] J. Mautz and R. Harrington, "Modal analysis of loaded n-port scatterers," IEEE Transactions on Antennas and Propagation, vol. 21, no. 2, pp. 188-199, March 1973.

[11] R. Harrington, "Reactively controlled directive arrays," IEEE Transactions on Antennas and Propagation, vol. 26, no. 3, pp. 390-395, May 1978.

[12] E. Newman and J. H. Richmond, "Superdirective receiving arrays," IEEE Transactions on Antennas and Propagation, vol. AP-26, no. 5, pp. 629635, September 1978.

[13] T. Ungan, M. Freunek, M. Muller, W. D. Walker, and L. M. Reindl, "Wireless energy transmission using electrically small antennas," in IEEE Radio and Wireless Symposium, 2009, pp. 526-529.

[14] N. Zhu, R. W. Ziolkowski, and H. Xin, "Electrically small gps 11 rectennas," IEEE Antennas and Wireless Propagation Letters, vol. 10 pp. 935-938, 2011.

[15] S. D. Assimonis, V. Fusco, A. Georgiadis, and T. Samaras, "Efficient and sensitive electrically small rectenna for ultra-low power if energy harvesting," Scientific Reports, vol. 8, no. 15038, pp. 1-13, October 2018.

[16] A. Georgiadis, A. Collado, and M. M. Tentzeris, Energy Harvesting: Technologies, Systems and Challenges. Cambridge University Press, 2021.

[17] A. Georgiadis, "A superdirective electrically small rectenna," in URSI General Assembly, Rome, Italy, 28 August - 4 September 2021, p. 1.

[18] D. Arceo and C. A. Balanis, "Design methodology for a reactively loaded yagi-uda antenna," IEEE Antennas and Wireless Propagation Letters, vol. 11, pp. 795-798, 2012.

[19] B. L. G. Jonsson, S. Shi, L. Wang, F. Ferrero, and L. Lizzi, "On methods to determine bounds on the $q$-factor for a given directivity," IEEE Transactions on Antennas and Propagation, vol. 65, no. 11, pp. 56865696, November 2017.

[20] J. H. Richmond, "Radiation and scattering by thin wire structures in the complex frequency domain," The Ohio State University, NASA CR-2396 TR 2902-10, May 1974.

[21] — - "Computer program for thin-wire structures in a homogeneous conducting medium," The Ohio State University, NASA CR-2399 TR 2902-12, June 1974.

[22] J. W. Eaton, D. Bateman, S. Hauberg, and R. Wehbring, GNU Octave version 6.1.0 manual: a high-level interactive language for numerical computations, 2020. [Online]. Available: https://www.gnu.org/software/octave/doc/v6.1.0/

[23] G. J. Burke, K. Miller, and A. J. Poggio, "The numerical electromagnetics code (NEC) - a brief history," in Proc. IEEE Antennas and Propagation Society Symposium, Monterey, vol. 3, 2004, pp. 2871-2874.

[24] (2018, May). [Online]. Available: http://www.w7ay.net/site/Applications/cocoaNEC/

[25] M. Gustafsson, D. Tayli, C. Ehrenborg, M. Cismasu, and S. Nordebo, "Antenna current optimization using matlab and cvx," Forum for Electromagnetic Research Methods and Application Technologies (FERMAT), vol. 15, no. 5, pp. 1-29, May-June 2016.

[26] J. Löfberg, "Yalmip : A toolbox for modeling and optimization in matlab," in In Proceedings of the CACSD Conference, 2004.

[27] J. F. Sturm, "Using sedumi 1.02, a matlab toolbox for optimization over symmetric cones," Optimization methods and software, vol. 11, no. 1-4, pp. 625-653, 1999.

[28] L. Vandenberghe and S. Boyd, "Semidefinite programming," SIAM Review, vol. 38, no. 1, pp. 49-95, March 1996.

[29] M. Fazel, H. Hindi, and S. P. Boyd, "A rank minimization heuristic with application to minimum order system approximation," in Proceedings of the 2001 American Control Conference. (Cat. No.01CH37148), vol. 6, 2001, pp. 4734-4739. 
[30] A. Mutapcic, S.-J. Kim, and S. Boyd, "Beamforming with uncertain weights," IEEE Signal Processing Letters, vol. 14, no. 5, pp. 348-351, May 2007.

[31] M. Margraf. (2021) QucsStudio - a free and powerful circuit simulator. [Online]. Available: http://dd6um.darc.de/QucsStudio/

[32] F. Bolos, J. Blanco, A. Collado, and A. Georgiadis, "Rf energy harvesting from multi-tone and digitally modulated signals," IEEE Transactions on Microwave Theory and Techniques, vol. 64, no. 6, pp. 1918-1927, June 2016.

[33] SKYWORKS, "Surface-mount mixer and detector schottky diodes," March 2021. 